Théologiques

Théologiques

\title{
Les approches féministes en bioéthique
}

\section{Susan Sherwin}

Volume 7, numéro 1, printemps 1999

Les trente ans de la bioéthique

URI : https://id.erudit.org/iderudit/024970ar

DOI : https://doi.org/10.7202/024970ar

Aller au sommaire du numéro

\section{Éditeur(s)}

Faculté de théologie de l'Université de Montréal

\section{ISSN}

1188-7109 (imprimé)

1492-1413 (numérique)

Découvrir la revue

\section{Citer cet article}

Sherwin, S. (1999). Les approches féministes en bioéthique. Théologiques, 7(1), 9-18. https://doi.org/10.7202/024970ar

\section{Résumé de l'article}

Les approches féministes en bioéthique permettent de mettre l'accent sur des réalités souvent laissées pour compte dans les discussions officielles. Une sensibilité particulière aux situations d'oppression et aux inégalités sociales ouvre la voie à des préoccupations qui élargissent les champs d'intérêt de la bioéthique et en questionnent les prises de position tant dans le domaine médical que social. La bioéthique féministe cherche à offrir des alternatives participatives là où règne souvent une hiérarchisation désengageante et dominatrice. 
Théologiques 7/1 (1999) 9-18.

\title{
Les approches féministes en bioéthique
}

\author{
Susan SHERWIN \\ Département de Philosophie \\ Université Dalhousie
}

L'intérêt tant académique que populaire pour les théories féministes s'est développé en même temps que l'intérêt pour la bioé thique. Dans les deux cas, l'intérêt remonte à la fin des années 60 et aux débuts des années 70 , même si certains événements importants de l'histoire de ces deux champs ont eu lieu avant ces dates (la publication en 1940 de Le deuxième sexe de Simone de Beauvoir et Le code de Nuremberg sur les recherches impliquant le sujet humain). Dès le début des années 70 , ces deux disciplines apparurent dans les revues et manuels scolaires, et les universités commencèrent à les enseigner. Depuis cette date, les écrits concernant ces deux champs d'intérêt se sont particulièrement multipliés.

Les champs féministe et bioéthique se ressemblent sous plusieurs aspects. Malgré une grande diversité de pensée et des approches différentes, ils possèdent un dénominateur commun. Parmi les différents points de vue qui caractérisent le féminisme, certains thèmes communs se retrouvent. Ces thèmes sont: un engagement pour les femmes; la reconnaissance que le genre (gender) joue un rôle important dans l'organisation de la plupart des sociétés; que la perception du rôle de la femme dans le domaine de la reproduction a été centrale pour leur statut social, économique et politique; et que les femmes sont injustement traitées dans la plupart (sinon toutes) les sociétés. De façon parallèle, différentes prises de position en bioéthique se sont caractérisées par un intérêt évident pour les questions éthiques qui appartiennent au domaine médical; malgré plusieurs points de divergence, il y a consensus pour que chaque patient soit traité avec respect et considération. Inutile d'ajouter qu'il y a place à différentes interprétations à l'intérieur de ces deux champs. 
Il y a eu, dès le début des débats en bioéthique, des théoriciens et praticiens qui ont étudié la bioéthique avec une approche féministe, de même que des féministes qui ont concentré leurs efforts sur les questions bioéthiques. Le croisement de ces deux disciplines est particulièrement visible lorsqu'il est question de la procréation (ex: avortement, contraception, et l'utilisation des méthodes reproduc tives). Les féministes ont demandé que les questions touchant la reproduction soulignent le fait que les femmes sont partiellement affectées par les politiques de reproduction pour des raisons biolo. giques et sociales : la période de grossesse et l'accouchement se produisent dans le corps de la femme, et cette dernière doit assumer des responsabilités disproportionnées quant à l'éducation des enfants.

Malgré cet intérêt, de part et d'autre, vis-à-vis une éthique des politiques de reproduction, l'engagement de poursuivre une approche féministe qui rejoigne toutes les dimensions de la bioéthique est assez récent. Ce n'est qu'en 1992 qu'apparurent les deux premiers livres traitant spécifiquement de la bioéthique féministe: Holmes et Purdy (1992) et Sherwin (1992). Depuis, les publications se sont multipliées. Récemment, les ouvrages de Purdy (1996), Wolf (1996), Tong (1997), Sherwin et all. (1998) et Donchin et Purdy (à venir).

La bioéthique féministe est apparue comme une branche à l'intérieur d'un champ plus large connu comme éthique féministe. Le terme «éthique féministe » réfère à n'importe quelle orientation éthique qui s'engage à inclure les questions et analyses féministes dans sa façon d'aborder les questions morales. Ce qui pourrait se traduire par une attention aux expériences et valeurs des femmes. Certaines théoriciennes vont identifier l'éthique féministe avec l'éthique du soin. Une telle interprétation rejoint ce qui est fondamental dans la préocuppation morale, à savoir la relation interpersonnelle. Ces mêmes théoriciennes rejettent les approches éthiques traditionnelles qui véhiculent des principes universels abstraits et impersonnels. Pour d'autres théoriciennes, comme moi, l'éthique féministe est caractérisée par le désir d'étudier les questions de domination et d'oppression (ainsi que de pouvoir et privilège). Contrairement aux théoriciens de l'éthique traditionnelle, qui évacuent les questions politiques du domaine éthique, ces féministes nous demandent de nous pencher sur les effets qu'une pratique ou 
politique vont avoir sur des modèles d'oppression existant quand ils concernent le statut moral.

En même temps qu'apparaît un débat au niveau de la théorie féministe et de la bioéthique, la bioéthique féministe concentre ses efforts afin d'introduire des perspectives féministes dans les débats éthiques qui surgissent à l'intérieur de la bioéthique. Le but de ces efforts est de modifier, et parfois d'initier, les débats de ces deux champs. Là où les non-féministes cherchent la neutralité des sexes dans les discussions, les féministes bioéthiques introduisent la perspective de l'analyse des sexes dans les thèmes abordés. Par ce fait, on cherche à enrichir les discussions abstraites d'une théorisation féministe, en réfléchissant sur la réalité concrète et la complexité des pratiques du système de santé.

Nous pouvons revoir quelques contributions majeures apportées par les bioéthiciennes féministes en examinant différents paliers du débat où la question de la bioéthique est abordée. Comme tous les bioéthiciens, les féministes cherchent à transformer le plus grand nombre de pratiques possibles. Encore plus, la plupart d'entre elles veulent développer des thèmes déjà proposés. D'autres apports incluent des efforts afin d'élargir le champ des perspectives considérées en bioéthique, ainsi que la proposition de réviser certains des termes et méthodes reliés aux activités bioéthiques. Proposons quelques exemples de chaque type.

La plus visible est la façon dont les théoriciennes féministes modifient les sujets familiers en bioéthique en introduisant les stratégies et concepts de l'éthique féministe. Par exemple, les féministes veulent s'assurer que les nouvelles propositions concernant le système de santé considèrent les nouveaux facteurs de la bioéthique. Ces facteurs mettent en relief les modèles d'oppression associés au genre (aussi la race, classe sociale, sexualité, l'âge et la déficience).

C'est dans le domaine des interventions médicales, en particulier les questions touchant la procréation, l'avortement et les techniques de reproduction, que l'engagement féministe est le plus important. Les approches non-féministes qui abordent ces questions ont tendance à les analyser à partir du principe de la sanctification de la vie humaine ou d'un engagement général envers l'autonomie 
personnelle; cette approche tente de résourdre les questions bioéthiques selon des principes abstraits et universels. Les débats féministes se rallient aux interprétations de la liberté de reproduction qui sont sensibles à une liberté sociale des femmes qui est en lien avec le contrôle, par les femmes, de la reproduction. La plupart des théori ciennes féministes refusent d'isoler les questions concernant l'avor. tement et les techniques de reproduction du contexte où elles surgissent. Elles insistent pour examiner les façons dont chaque pratique affecte les rôles et statuts des femmes dans la société en considérant que certains groupes de femmes peuvent être affectés différemment.

Les perspectives féministes sont aussi présentes dans les recherches cliniques sur l'être humain. Dans la littérature nonféministe, il y a un consensus à propos du besoin de règles éthiques qui protègeraient les individus de l'exploitation et de l'abus. Un intérêt particulier vise les groupes plus vulnérables comme les enfants, les personnes en institution, et celles avec des déficiences. Les éthiciennes féministes soulignent qu'être membre d'un groupe oppressé et socialement dévalué peut rendre une personne sujette à l'exploitation, et que des règles explicites devraient protéger ces groupes de l'abus expérimental. En même temps, la plupart des féministes refusent le modèle historique qui oriente la recherche thérapeutique vers les membres privilégiés de la société (habituellement les hommes blancs) en ignorant les besoins de santé des membres des autres groupes. Cet agenda de recherche laisse souvent les intervenants de la santé avec des données insuffisantes pour prendre les décisions au nom des patients des groupes négligés.

De la même manière, les théoriciennes féministes demandent que la liste des préoccupations débordent jusque dans le domaine complexe de l'euthanasie et des médecins-assistants au suicide. La prise de conscience féministe soulève les différentes façons dont les femmes peuvent être affectées lorsque les politiques sont instaurées. Par exemple, les politiques qui empêchent les personnes gravement malades de mettre fin à leurs jours doivent admettre que ces personnes requièrent des soins particuliers. Malheureusement, le soin de ces personnes retombe sur les épaules des travailleuses sociales et des membres féminins de la famille; c'est-à-dire que le choix de prolonger ces vies devient un fardeau additionnel pour plusieurs femmes. En même temps, les féministes sont particulièrement conscientes du 
fait que les vies des opprimés sont dévaluées dans la société, ce qui les rend vulnérables à toute politique libérale d'euthanasie. En face d'une telle complexité, un consensus entre féministes n'est pas encore atteint.

Cependant, la bioéthique féministe n'est pas restreinte aux questions de morale pré-établies. Elle peut être définie par un désir d'élargir la liste des questions qui nécessitent d'être abordées à l'intérieur du champ de la bioéthique. Par exemple, les normes qui guident la pratique de la chirurgie plastique. Cette forme de chirurgie, dispendieuse et risquée, cherche à rendre les femmes conformes aux normes artificielles et élusives de la beauté féminine. Les arguments habituels qui sous-tendent le consentement à de telles chirurgies sont insensibles aux effets, compromettant une autonomie qui est affectée par une culture qui continue de dire aux femmes que les signes de l'âge et de la grosseur sont intolérables. Les réflexions rationnelles semblent être de pauvres arguments pour l'autonomie, quand des conditions oppressives exigent que des femmes échangent leurs chances de succès au travail ou en amour contre leur propre santé. Jusqu'à présent, seules les bioéthiciennes féministes ont considéré ce sujet digne d'un débat sérieux.

De façon plus large, le féminisme nous aide à voir la nécessité de questionner les théories du corps abordées selon les perspectives médicales. Le point de vue scientifique ou médical du corps a une tendance aliénante de soigner le corps et ses parties de façon séparée de la personne qui les "habite». La médecine nous enseigne que le corps doit être objet de surveillance : vérifier régulièrement les signes « envahisseurs " (invasion) ou "de rupture " (breakdown) et les faire diagnostiquer, par les experts, à intervalles réguliers. Parce que les autorités médicales sont accréditées pour connaître nos corps mieux que nous-mêmes, nous sommes en état de dépendance face à ces autorités. Leur autorité est socialement renforcée de maintes façons : elle est l'ultime arbitre dans les cas de congé de maladie, d'assurance-maladie et autres bénéfices. Les bioéthiciennes féministes voient comme un débat sérieux et une problématique morale ces questions d'épistémologie et de pouvoir social.

D'autres problématiques deviennent évidentes lorsqu'elles sont envisagées par la perspective de la bioéthique féministe. Par 
exemple, l'oppression implicite qui gouverne les normes traitant les déficiences a incité les théoriciennes à développer des théories féministes sur le sujet (Wendell 1996). D'autres ont relancé le débat de la "génétisation" (geneticization) des problèmes sociaux (Lippman dans Sherwin et all. 1998). Et d'autres féministes insistent pour que nous examinions la structure hiérarchique du système de santé (Sherwin 1992).

En plus de vouloir élargir le calendrier bioéthique déjà existant, la bioéthique féministe encourage le développement des perspectives engagées. Elles ont été particulièrement critiques envers une littérature bioéthique traditionnelle qui aborde exlusivement les problèmes éthiques soulevés par des médecins et des administrateurs de la santé. Par contraste, les féministes essayent d'aborder les questions du point de vue des personnes moins privilégiées, comme les patients, les autres membres de l'équipe des soins, amis et famille des patients, et ceux qui sont à l'écart du système d'aide à la santé (comme ceux qui manquent de ressources financières pour les soins de santé tandis que certains fonds sont dirigés vers des services spécialisés pour les privilégiés). L'éthique féministe valorise les discernements de ces différentes perspectives et cherche à élargir les discussions bioéthiques de façon substantielle.

Par ailleurs, il y a une littérature bioéthique marginale associée au domaine de la santé autre que la médecine. Cette littérature est développée par le personnel soignant. L'éthique du personnel soignant met en lumière la spécificité de leur travail, distincte de celle des médecins, en soulignant que l'attention est portée davantage au soin (caring) plutôt que de guérir, et que cette profession est largement attribuée aux femmes. Il n'est pas surprenant que l'éthique du soin ait été bienvenue dans le monde de l'éthique. Cependant, le travail en éthique du soin n'est pas seulement féministe, car son analyse n'a pas de genre spécifique et son attention ne porte pas sur les autres formes d'oppression. La reconnaisance d'un apport féminin distinct dans ce domaine continue de perpétuer des stéréotypes concernant les normes sexuelles (et d'oppression). Une éthique du soin est féministe lorsqu'elle ne valorise pas l'engagement théorique face à la responsabilité traditionnelle de la femme tant quant aux soins, mais en critique plutôt les attentes. 
En général, l'approche féministe en bioéthique rejette la hiérarchie traditionnelle de la structure médicale. En particulier, elle cherche à promouvoir des structures qui permettent aux patients d'être des participants actifs dans la pratique des soins - incluant les pratiques bioéthiques - avec un contrôle sur le choix des services disponibles et la manière dont ils sont dispensés. Par exemple, les féministes demandent de nouvelles définitions quant aux normes médicales de la santé, de la maladie, de la reproduction, et proposent à la médecine un rôle modeste de support et de promotion de la santé.

Le féminisme nous incite à poser la question de la tendance médicale à définir et poser les problèmes de santé comme relevant entièrement de l'individu. Le modèle bio-médical de la santé juge que l'individu est le meilleur lieu pour parler de la maladie et des déficiences. Il sera toujours nécessaire de fournir des services de santé aux individus, cependant, bien des féministes insistent pour qu'une politique de la santé rejoigne une perspective plus large. Les effets de la violence domestique, des grossesses non-voulues et la malnutrition due à la pauvreté nécessitent une intervention appropriée à chaque situation; ces situations requièrent la fin des pratiques injustes et des changements dans les attitudes sociales.

Le médecin nous encourage à voir le côté personnel et non la dimension politique à laquelle sont confrontés les patients aux prises avec des difficultés d'ordre psychiatrique, de dépendance, d'infertilité, de déficience, de cancer, ou d'âge avancé. Habituellement, la bioéthique reproduit l'approche médicale en demandant ce que le patient veut afin de trouver le soin médical approprié. Par contraste, la bioéthique féministe pose des questions à propos du contexte dans lequel s'insère le problème identifié : les questions d'ordre social et politique peuvent être essentielles pour trouver l'approche médicale et bioéthique adéquate à une situation particulière. Par exemple, le cancer du sein ne devrait pas être envisagé comme une maladie tragique qui affecte des millions de femmes, mais plutôt comme une réalité qui a dramatiquement augmenté. Une telle réalité nous invite à poser la question du rôle de l'environnement dans le développement de cette maladie. Au-delà de la recherche d'un traitement, ce sont les stratégies politiques qui sont visées. 
Encore plus, la bioéthique féministe introduit souvent de nouveaux instruments méthodologiques et conceptuels. Méthodologiquement, bien des féministes rejettent les distinctions entre fait et valeur, entre théorie et pratique; ces distinctions peuvent être perçues comme rejettant et traversant les barrières artificielles entre la théorisation académique et l'activité sociale.

Une ré-interprétation des concepts-clés en bioéthique est aussi requise. Par exemple, les féministes sont particulièrement inconfortables avec le terme "patient ", car son sens métaphorique suggère la passivité et la dépendance; son usage perpétue l'inacceptable assomption à propos de la nature de la relation entre les intervenants de la santé et ceux qui demandent des soins. Des substitutions comme " consommateur" (consumer) ou "client " (client) soulèvent des pro blèmes d'interprétation rattachés au marché du travail, qui peuvent aussi affecter les relations avec les intervenants de la santé. L'effort féministe en éthique relationnelle promet de fournir un répertoire de modèles pour différentes sortes de relations entre lesdits patients et les intervenants de la santé.

Plusieurs féministes ont proposé différentes interprétations et applications du principe de justice qui se trouve au centre de plusieurs débats concernant les soins de santé (ex. Nelson et Nelson dans Wolf 1996). Le féminisme soulève le besoin d'aller au-delà de l'interprétation du concept de justice afin de rejoindre tous les aspects d'injustice inhérents à l'oppression. Les féministes s'opposent à la tendance des bioéthiciennes non-féministes qui réduisent les questions de justice en matière de santé à une distribution équitable des ressources médicales. Une telle approche rend obscures les autres formes d'injustices qui touchent les membres de la société qui sont vulnérables à la maladie. Les analyses féministes nous rappellent que le rôle de la pauvreté est un indicateur au niveau de la santé, soulignant que cette dernière est largement la condition des femmes et des enfants dans les minorités visibles, des personnes déficientes et âgées. Les discussions bioéthiques concernant les politiques des soins de la santé devraient être plus sensibles aux questions de sexe, race, âge et statut social. La justice requiert non seulement une répartition équitable des lits dans les unités intensives ( comme c'est le cas dans les discussions traditionnelles) mais doit promouvoir, en premier lieu, la santé. Des changements devraient inclure, par exemple, de 
la nourriture et un logement adéquat, un environnement protégé contre les effets toxiques et la violence, le développement de l'estime de soi, et l'accès à une éducation de la santé. Cette orientation implique un déplacement des visées actuelles des services de la santé et de la bioéthique, qui s'éloigne des interventions évasives et technologiques pour se rapprocher des stratégies qui visent premièrement la santé.

Les théoriciennes féministes soulèvent le besoin de réviser l'interprétation traditionnelle du concept d'autonomie. Soulignant le fait que certaines formules traditionnelles ignorent les effets subtils des conditions sociales oppressives, plusieurs féministes rejettent la tendance individualiste qui sous-tend les formules les plus communes de l'autonomie. Pendant que certaines féministes préviennent l'usage d'un tel concept, d'autres prônent une ré-interprétation à l'aide d'une approche relationnelle sensible (Sherwin et all. 1998). Elles suggèrent que nous allions au-delà des efforts qui évaluent la compétence de l'autonomie de certains patients (bioéthique traditionnelle) et commencions à considérer les différentes façons dont la société promeut et maîtrise l'autonomie de certains groupes. Le respect du patient requiert non seulement une information concernant des procédures spécifiques, mais aussi des efforts pour abolir les barrières oppressives qui empêchent l'autonomie.

En somme, la bioéthique féministe apporte une contribution au vaste champ de la bioéthique en y introduisant la pensée féministe. Elle transforme le contenu du sujet et de tous les niveaux de l'analyse. En insistant sur la pertinence des dimensions sociales et politiques des problèmes étudiés, les bioéthiciennnes féministes enrichissent et approfondissent les discussions bioéthiques de plusieurs façons.

\section{Bibliographie}

DONCHIN, A. et L.M. PURDY, Embodying Bioethics, Recent Feminist Advances. Lantham MD, Rowman \& Littlefield, 1999.

HOLMES, H.B. et L.M. PURDY eds, Feminist Perspectives in Medical Ethics. Bloomington, Indiana University Press, 1992. 
PURDY, L., Reproducting Persons. Issues in Feminist Bioethics. Ithaca, NY, Cornell University Press, 1996.

SHERWIN, S., No longer Patient. Feminist Ethics and Health Care. Philadelphia, Temple University Press, 1992.

SHERWIN, S. Coordinator, "The Feminist Health Care Ethics Research Network", dans The Politics of Women's Health. Exploring Agency and Autonomy. Philadelphia, Temple University Press, 1998.

TONG, R., Feminist Approches to Bioethics. Theoretical Reflections and Practical Applications. Boulder, CO, Westview Press, 1997.

WENDELL, S., The Rejected Body. Feminist Philosophical Reflections on Disability. New York, NY, Routledge, 1996.

WOLF, S. ed., Feminism $\mathcal{E}$ Bioethics. Beyond Reproduction. New York, Oxford University Press, 1996. 